\title{
Early Onset Polymorphic Post-transplant Lymphoproliferative Disease Mimicking a Solitary Necrotizing Abscess in a Graft Liver
}

\author{
Pil Soo Sung ${ }^{1 *}$, Jaejun Lee ${ }^{1 *}$, Joon Lee ${ }^{2 *}$, Hee Chul Nam², Si Hyun Bae', Seung Kew Yoon ${ }^{2}$ \\ 'Department of Internal Medicine, Eunpyeong St. Mary's Hospital, Seoul; 'Department of Internal Medicine, Seoul St. Mary's Hospital, \\ College of Medicine, The Catholic University of Korea, Seoul, Korea
}

Received May 22, 2019

Revised Aug. 7, 2019

Accepted Aug. 23, 2019
Although post-transplantation lymphoproliferative disease (PTLD) after liver transplantation is very rare, its prognosis is worse than that of PTLD following other types of solid organ transplantation. Here, we report a rare case of early onset polymorphic PTLD in a graft liver occurring five months after deceased-donor liver transplantation due to hepatocellular carcinoma and hepatitis C virus infection. Initially, findings from contrast-enhanced magnetic resonance imaging mistakenly suspected the lesion was a necrotizing abscess with central necrosis. However, ${ }^{18} \mathrm{~F}$-fluorodeoxyglucose positron emission tomography and biopsy findings confirmed an Epstein-Barr virus (EBV)-associated, B cell type polymorphic PTLD with central necrosis. Our case suggests regular monitoring of EBV serologic status for liver transplant recipients who were initially in an EBV seronegative state. Although early-onset PTLD is very rare after liver transplantation, PTLD should be suspected when recipients show the seroconversion for EBV proteins and the development of new tumors with various clinical presentations. (J Liver Cancer 2019;19:165-170)

Keywords: Post-transplantation lymphoproliferative disease; Epstein-Barr virus; ${ }^{18} \mathrm{~F}$-fluorodeoxyglucose positron emission tomography

\section{INTRODUCTION}

Post-transplantation lymphoproliferative disease (PTLD) is one of the most common neoplastic diseases in recipients of solid organ transplantation (SOT). ${ }^{1,2}$ Previous reports have demonstrated that approximately 70\% of PTLD cases are associated with an Epstein-Barr virus (EBV) infection and the use of immunosuppressive drugs. ${ }^{2}$ A mismatch between EBV seropositive donor and EBV seronegative recipient can result

\section{Corresponding author : Seung Kew Yoon}

Department of Internal Medicine, Seoul St. Mary's Hospital, 222 Banpodaero, Seocho-gu, Seoul 06591, Korea

Tel. +82-2-2258-2073, Fax. +82-2-3481-4025

E-mail; yoonsk@catholic.ac.kr

https://orcid.org/0000-0002-4476-4868 in the development of PTLD with a 4.7-fold higher hazard ratio compared to the matched EBV seropositive recipients. ${ }^{2}$ Though the exact pathogenesis is unclear, EBV-associated PTLD may be caused by the decrease in EBV-specific cytotoxic T-cells in recipients and the proliferation of EBV-infected B cells from the donor. ${ }^{3}$ Life-long intake of immunosuppressive agents increases the incidence of PTLD after liver transplantation. Individual medications that are reported as potential risk factors for PTLD are anti-thymocyte globulin, calcineurin inhibitors, anti-CD3, tacrolimus, and cyclosporine. $^{2,4-7}$

Approximately half of the cases of reported PTLD occur in transplanted organs, such as the heart, lung, and liver. ${ }^{6,8}$ As the survival rates for SOT patients have increased recently, the majority of PTLD cases are late onset cases. ${ }^{9}$ Early onset

*These three authors contributed equally to this work. 
PTLD, developing within the first six months after transplantation, rarely occurs after liver transplantation. ${ }^{10}$ Here, we report an early-onset EBV-associated polymorphic PTLD in a graft liver, which was initially suspected to be a necrotizing abscess with central necrosis by contrast-enhanced abdominal magnetic resonance imaging (MRI), but diagnosed as polymorphic PTLD after ${ }^{18} \mathrm{~F}$-fluorodeoxyglucose positron emission tomography $\left({ }^{18} \mathrm{~F}\right.$-FDG PET $)$ and pathologic findings.

\section{CASE REPORT}

\section{History and presentation}

The present case report was approved by the Institutional Review Boards (Seoul St. Mary's Hospital, KC19ZESI0149). A 54-year-old male patient was admitted for the evaluation of a hepatic mass. The patient had undergone deceased-donor liver transplantation (LT) for hepatocellular carcinoma (HCC) (Modified UICC 5th stage II, T2N0M0, Barcelona Clinic Liver Cancer stage: A) due to chronic hepatitis C virus (HCV) infection. The patient had been diagnosed with HCC three years prior to liver transplantation and had undergone trans-arterial chemoembolization nine times. At the time of liver transplantation, the viable tumor was $1 \mathrm{~cm}$ in size. After surgery, the patient was prescribed $400 \mathrm{mg}$ cyclosporin, 1,000 mg mycophenolate mofetil, and $5 \mathrm{mg}$ prednisolone daily. Five months later, the patient was admitted to the hospital for a follow-up examination during a state of complete remission of HCC with no specific symptoms.

\section{Investigations}

Investigations revealed normal ranges of complete blood count, blood chemistry, and tumor markers and elevated serum lactate dehydrogenase levels $(456 \mu \mathrm{g} / \mathrm{dL})$. HCV RNA level was 5,452,120 IU/mL indicating a recurrence of $\mathrm{HCV}$. Contrast-enhanced abdominal MRI detected a $7 \mathrm{~cm}$ irregular necrotizing mass with rim enhancement in the right hemiliver mimicking a necrotizing abscess (Fig. 1A). ${ }^{18}$ F-FDG PET showed a single hypermetabolic malignant tumor with central hypometabolism (Fig. 1B). Ultrasound-guided biopsy of the mass was performed for diagnosis. Biopsy of the mass demonstrated a polymorphic PTLD with central necrosis (Fig. 2A). The malignant cells showed a B cell phenotype with plasmocytic differentiation (CD20, CD38 and IgG positive, kappa light chain restricted) (Fig. 2B, C). Most of the malignant cells were positive for Epstein-Barr encoding region (EBER) in situ hybridization (Fig. 2D). Serum EBV DNA level was $20,368 \mathrm{IU} / \mathrm{mL}$, which had been negative be-
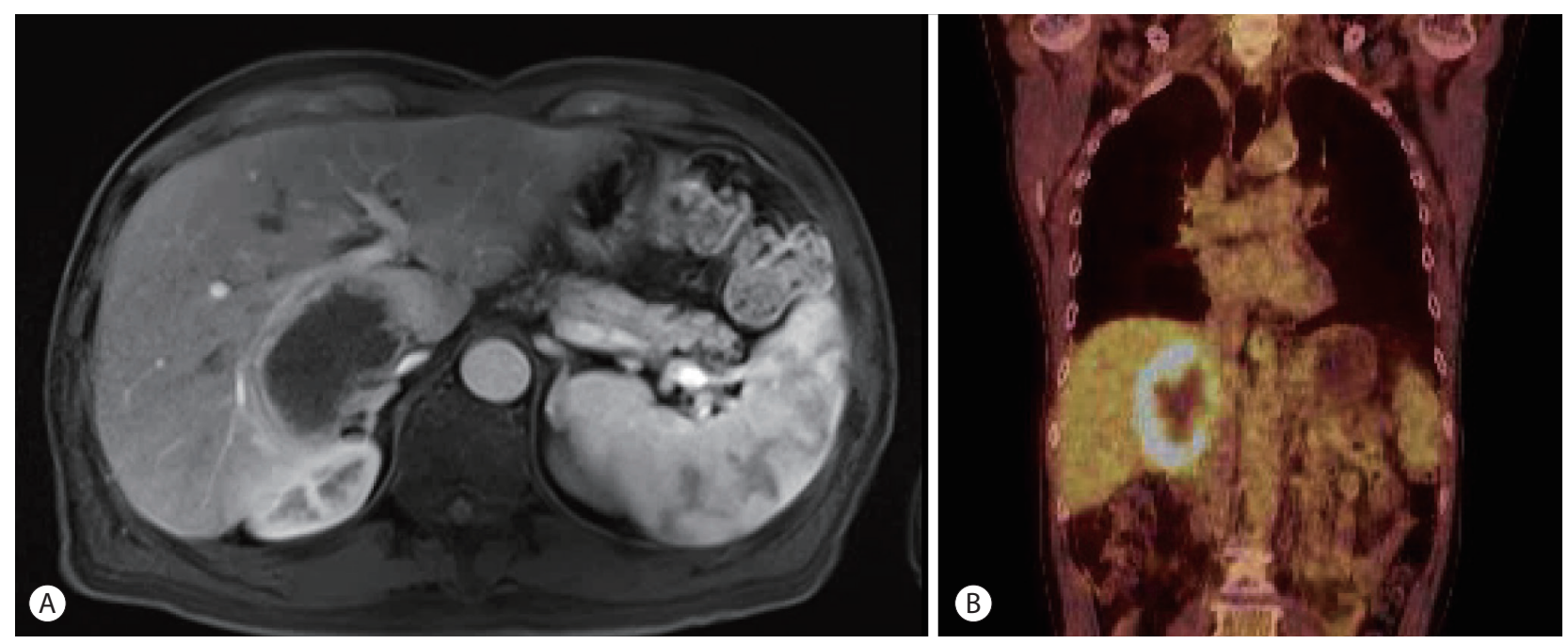

Figure 1. Radiologic findings of the necrotizing mass in the graft liver. (A) Contrast-enhanced abdominal magnetic resonance imaging (portal phase) detected a $7 \mathrm{~cm}$ irregular necrotizing mass with rim enhancement in the right hemi-liver mimicking a necrotizing abscess. (B) ${ }^{18} \mathrm{~F}$-fluorodeoxyglucose positron emission tomography shows a single hypermetabolic malignant tumor with central hypometabolism. 

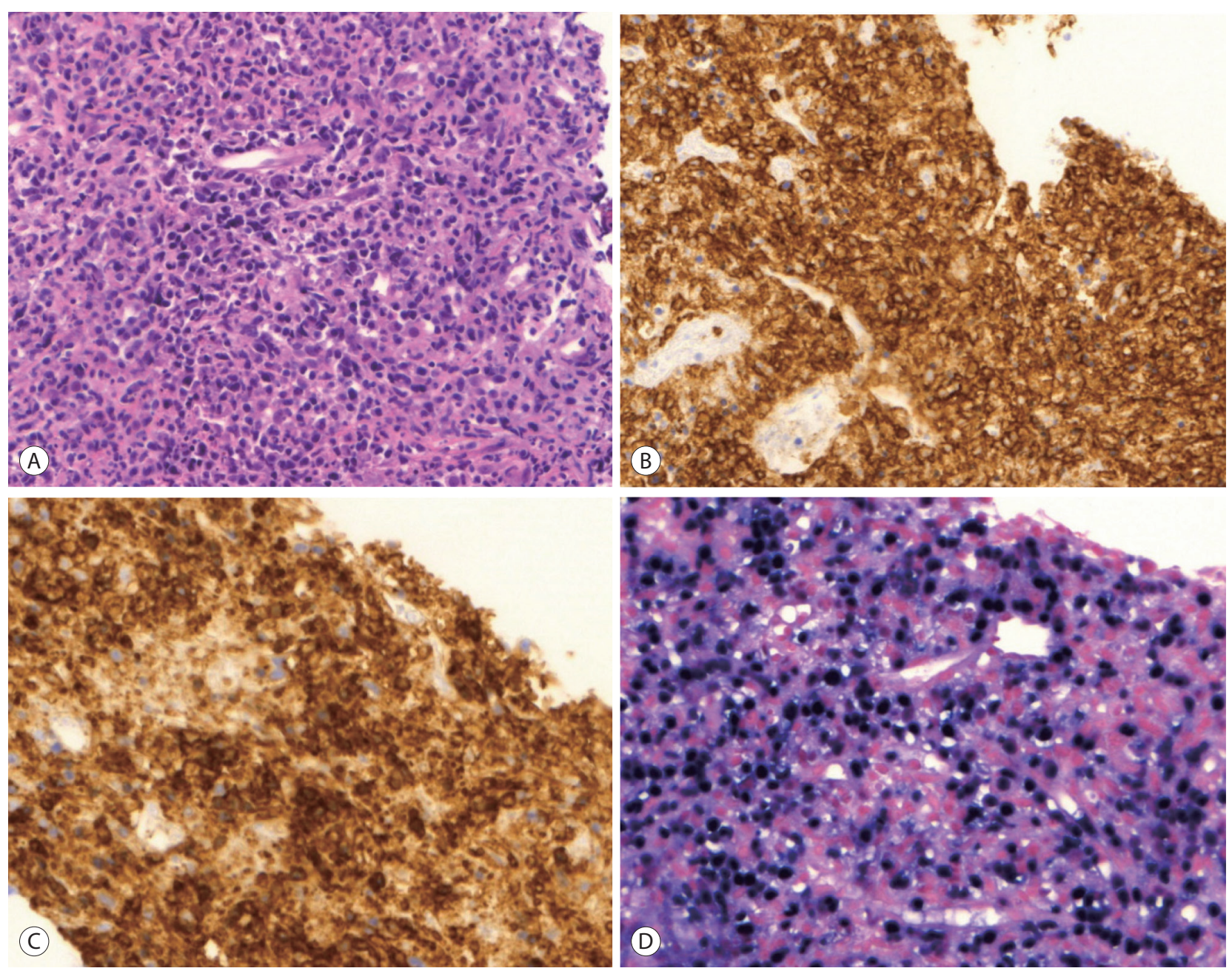

Figure 2. Pathologic findings of the necrotizing mass in the graft liver (original magnification $\times 200$ ). (A) H\&E staining demonstrates a polymorphic post-transplantation lymphoproliferative disease with central necrosis. (B) Immunohistochemistry of CD20 shows positive cytoplasmic staining. (C) Immunohistochemistry of CD38 shows positive cytoplasmic staining. (D) In situ hybridization of Epstein-Barr-encoding region of RNA shows that most of the malignant cells are positive for the hybridization.

fore liver transplantation. A retrospective review of the blood test results of the deceased donor revealed that the donor was seropositive for the viral capsid antigen of EBV. A bone marrow biopsy was performed for the patient, and findings were normal. The international prognostic index was calculated and found to be one point (low-risk) and Ann Arbor staging was evaluated as stage I (single lymph node region or one extra-lymphatic site involvement).

\section{Treatment and outcome}

The patient underwent direct-acting antiviral treatment (le- dipasvir, sofosbuvir, and ribavirin) for recurrent HCV infection while simultaneously discontinuing cyclosporin and mycophenolate mofetil. As a substitute for immunosuppressants, $2.5 \mathrm{mg}$ everolimus was applied. After completion of the antiHCV therapy, chemotherapy was started. Four cycles of $600 \mathrm{mg}$ rituximab were administered every four weeks, which resulted in a decrease in the PTLD lesion to $5 \mathrm{~cm}$ in contrast enhanced computed tomography (CT) imaging. Eight sequential cycles of rituximab, cyclophosphamide, doxorubicin, vincristine, and prednisolone (R-CHOP) were administered in 6 months without prednisolone because of a history of gastric ulcer bleeding. After the completion of chemotherapy, response evaluation 


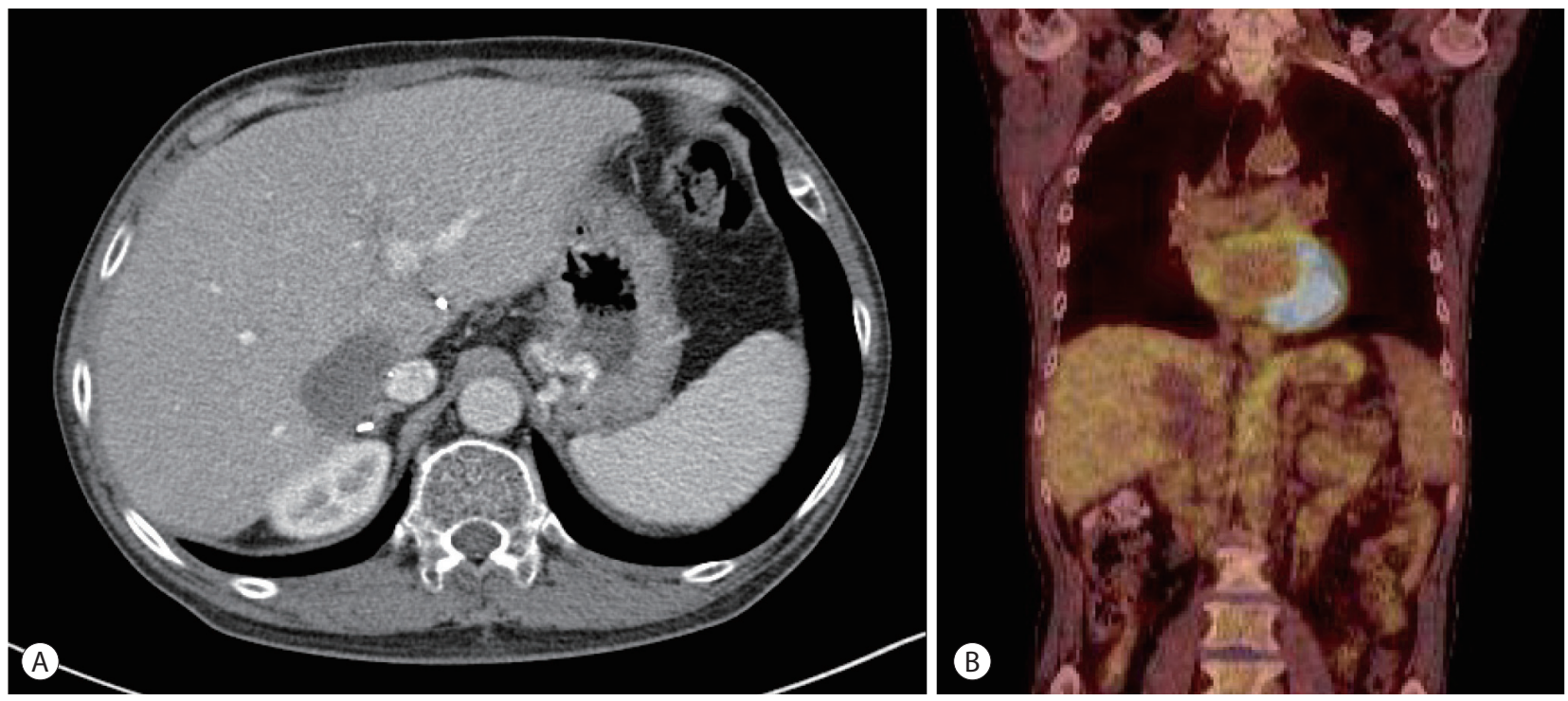

Figure 3. Follow-up imaging findings after treatment. (A) Contrast-enhanced abdominal computed tomography imaging of the graft liver shows a dramatic decrease in the post-transplantation lymphoproliferative disease (PTLD) lesion after treatment. (B) ${ }^{18} \mathrm{~F}$-fluorodeoxyglucose $\left({ }^{18} \mathrm{~F}\right.$-FDG) positron emission tomography imaging reveals no visible ${ }^{18} \mathrm{~F}-\mathrm{FDG}$ uptake in the PTLD lesion after treatment.

with contrast enhanced CT imaging and ${ }^{18}$ F-FDG PET revealed a further decrease in PTLD lesion and no visible ${ }^{18} \mathrm{~F}$-FDG uptake (Fig. 3). At this time, EBV-DNA was not detected.

\section{DISCUSSION}

The highest incidence of PTLD following SOT occurs in intestinal and multi-organ transplants (5-20\%), and the lowest incidence occurs in kidney and liver transplants (1-5\%). ${ }^{6}$ In comparison to other organ transplantations with prolonged use of immunosuppressants, PTLD associated with liver or kidney transplants occurs at a lower rate due to the reduced dose and administration duration of immunosuppressants. ${ }^{11}$ Previous reports demonstrated that PTLD after liver transplantation appeared most commonly in liver grafts (approximately 20\%). ${ }^{12}$

PTLD following liver transplantation typically occurs more than one year after the transplant in adults, because this is the period where patients receive long-term immunosuppressant treatment. ${ }^{10}$ Generally, $60-80 \%$ of all PTLD cases are EBV-related, ${ }^{13}$ and comprehensive analysis of PTLD cases after liver transplantation demonstrated that EBV-related cases account for $80 \%$ of all PTLD cases after transplanta- tion. Interestingly, $91 \%$ of early-onset PTLD cases were associated with EBV infection, although only $66 \%$ of late-onset PTLD cases were EBV-related. ${ }^{14}$ Since EBV is a $\gamma$-herpesvirus that stimulates B cell proliferation and transformation, $85 \%$ of PTLD cases are B-lymphocytic in origin. ${ }^{14}$ After successful treatment of the primary infection, EBV persists in infected $B$ cells and establishes a latent infection. Pharmacologic immunosuppression after transplantation results in the decreased frequency and functional impairment of EBV-specific cytotoxic T-cells. ${ }^{12}$ In this setting, EBV-driven lymphoproliferation with type 3 latency may occur and is labeled PTLD. ${ }^{12}$

$\mathrm{HCV}$ infection has also been reported as a possible risk factor for non-Hodgkin's lymphoma, especially for B cell lymphoma. ${ }^{15}$ The possible pathophysiology of HCV infection and PTLD suggests that chronic stimulation of the immune system because of HCV infection may cause clonal B cell expansions and lymphoproliferative conditions. ${ }^{16}$ However, findings from multiple studies indicate that the role of $\mathrm{HCV}$ as a risk factor for PTLD remains controversial. A retrospective cohort study for SOT patients with diagnosed PTLD during follow-up examination reported that HCV was not a major risk for PTLD in both liver and non-liver transplantations. $^{16}$ 
The diagnosis of PTLD following liver transplantation depends upon the findings from a liver biopsy. Classification of PTLD requires immunophenotyping for assessing light chain class restriction and lymphoid subsets, histopathology, and EBER in situ hybridization. ${ }^{17}$ Based on the pathological findings, the World Health Organization classifies PTLD into four categories: 1) early lesions (plasmocytic hyperplasia, infectious mononucleosis-like PTLD), 2) polymorphic PTLD, 3) monomorphic PTLD (B cell neoplasms, T-cell neoplasms), and 4) classical Hodgkin's lymphoma-type PTLD. ${ }^{17}$ Polymorphic PTLD, as seen in the present case, involves varying differentiation stages. As presented in this patient, the infiltrate causes the underlying tissue architecture to be effaced and destructed with malignant features, such as necrosis and high mitotic rate with nuclear atypia. ${ }^{18}$ Moreover, imaging is often helpful for the diagnosis of PTLD, and ${ }^{18} \mathrm{~F}$ FDG PET has been considered more sensitive than contrastenhanced CT and MRI. ${ }^{18}$ In this case, contrast-enhanced MRI image findings coincided with the interpretation of the lesion as a necrotizing abscess due to low attenuation and signal at the center of the lesion with rim enhancement. However, ${ }^{18} \mathrm{~F}$-FDG PET findings showed intense ${ }^{18} \mathrm{~F}$-FDG uptake at the margins of the lesion, suggesting a strong possibility of PTLD with central necrosis.

Although there is no standard treatment approved for the management of PTLD due to its clinical and pathological heterogeneity, the initial treatment typically involves a reduction in immunosuppression and a renewal of immune surveillance. The restoration of immune function allows the body to control EBV-infected proliferating cells. ${ }^{6}$ Both European and American guidelines also suggest a reduction in anti-proliferative agents such as mycophenolate mofetil and azathioprine, and switching to a mammalian target of rapamycin inhibitor, such as everolimus in this case. ${ }^{3}$ In the cases of CD20-positive PTLD with extrahepatic invasion or resistance to immunosuppressant reduction, rituximab is considered as the first-line treatment. ${ }^{19}$ The treatment response of first-line rituximab appeared to be $40-65 \% .{ }^{19}$ Early introduction of rituximab in patients with PTLD is reported to confer better survival outcomes. ${ }^{20}$ In cases of rituximabresistant PTLD or high-grade lymphoma or multi-organ in- volved PTLD, R-CHOP is recommended. ${ }^{6}$ For CD20-positive PTLD patients with rituximab-included regimen resistance, cytotoxic chemotherapy is considered a possible modality; however, the overall prognosis appeared to be worse when compared to that with rituximab-responsive PTLD. ${ }^{4}$ Other treatments such as surgical resection and radiotherapy for local control may be recommended for patients with resistance to the first-line treatment, but they may carry adverse side effects. ${ }^{4}$

Prognosis of PTLD following SOT is poor with a $60 \%$ and $40 \% 3$-year and 5-year overall survival rate, respectively. ${ }^{20}$ Hence, PTLD in SOT recipients should be promptly suspected based on clinical presentations and EBV serological state. However, it is difficult to reliably diagnose early-onset PTLD and initiate treatment due to the heterogeneity of clinical presentations. In conclusion, polymorphic PTLD in liver transplant recipients, less than one year after the transplantation, is rare but can cause fatal complications. Patients should undergo regular follow-up examinations for EBV serologic status. Atypical radiologic presentations, as in this case, should not be overlooked and should be carefully investigated for the possible occurrence of early-onset PTLD.

\section{ACKNOWLEDGEMENTS}

This work was supported by the research fund of Seoul St. Mary's Hospital (S.P.S.). The present case report was approved by the Institutional Review Boards (Seoul St. Mary's Hospital, KC19ZESI0149).

\section{Conflicts of Interest}

The authors have no conflicts to disclose.

\section{REFERENCES}

1. Jiang Y, Villeneuve PJ, Fenton SS, Schaubel DE, Lilly L, Mao Y. Liver transplantation and subsequent risk of cancer: findings from a Canadian cohort study. Liver Transpl 2008;14:1588-1597.

2. Dierickx D, Cardinaels N. Posttransplant lymphoproliferative disorders following liver transplantation: where are we now? World J Gastroenterol 2015;21:11034-11043.

3. Hatton O, Martinez OM, Esquivel CO. Emerging therapeutic strate- 
gies for Epstein-Barr virus+ post-transplant lymphoproliferative disorder. Pediatr Transplant 2012;16:220-229.

4. Dierickx D, Tousseyn T, Gheysens O. How I treat posttransplant lymphoproliferative disorders. Blood 2015;126:2274-2283.

5. Lauro A, Arpinati M, Pinna AD. Managing the challenge of PTLD in liver and bowel transplant recipients. Br J Haematol 2015;169:157172.

6. Petrara MR, Giunco S, Serraino D, Dolcetti R, De Rossi A. Posttransplant lymphoproliferative disorders: from epidemiology to pathogenesis-driven treatment. Cancer Lett 2015;369:37-44.

7. Kim H, Yi NJ, Lee J, Kim J, Moon MR, Jeong J, et al. Safety of reduced dose of mycophenolate mofetil combined with tacrolimus in living-donor liver transplantation. Clin Mol Hepatol 2014;20:291299.

8. Jagadeesh D, Woda BA, Draper J, Evens AM. Post transplant lymphoproliferative disorders: risk, classification, and therapeutic recommendations. Curr Treat Options Oncol 2012;13:122-136.

9. Jain A, Nalesnik M, Reyes J, Pokharna R, Mazariegos G, Green M, et al. Posttransplant lymphoproliferative disorders in liver transplantation: a 20-year experience. Ann Surg 2002;236:429-436; discussion 436-437.

10. Leblond V, Choquet S. Lymphoproliferative disorders after liver transplantation. J Hepatol 2004;40:728-735.

11. Al-Mansour Z, Nelson BP, Evens AM. Post-transplant lymphoproliferative disease (PTLD): risk factors, diagnosis, and current treatment strategies. Curr Hematol Malig Rep 2013;8:173-183.

12. Kamdar KY, Rooney CM, Heslop HE. Posttransplant lymphoproliferative disease following liver transplantation. Curr Opin Organ Transplant 2011;16:274-280.

13. Yoon SO, Yu E, Cho YM, Suh C, Kim KM, Han DJ, et al. Posttransplant lymphoproliferative disorders: clinicopathological analysis of 43 cases in a single center, 1990-2009. Clin Transplant 2012;26:67-73.

14. Izadi M, Taheri S. Features, predictors and prognosis of lymphoproliferative disorders post-liver transplantation regarding disease presentation time: report from the PTLD. Int. survey. Ann Transplant 2011;16:39-47.

15. Nieters A, Kallinowski B, Brennan P, Ott M, Maynadié M, Benavente $Y$, et al. Hepatitis $C$ and risk of lymphoma: results of the European multicenter case-control study EPILYMPH. Gastroenterology 2006;131:1879-1886.

16. Morton LM, Landgren O, Chatterjee N, Castenson D, Parsons R, Hoover RN, et al. Hepatitis C virus infection and risk of posttransplantation lymphoproliferative disorder among solid organ transplant recipients. Blood 2007;110:4599-4605.

17. Cazzola M. Introduction to a review series: the 2016 revision of the WHO classification of tumors of hematopoietic and lymphoid tissues. Blood 2016;127:2361-2364.

18. Parker A, Bowles K, Bradley JA, Emery V, Featherstone C, Gupte G, et al. Diagnosis of post-transplant lymphoproliferative disorder in solid organ transplant recipients - BCSH and BTS Guidelines. Br J Haematol 2010;149:675-692.

19. Martínez-Calle N, Alfonso A, Rifón J, Herrero I, Errasti P, Rábago $G$, et al. First-line use of rituximab correlates with increased overall survival in late post-transplant lymphoproliferative disorders: retrospective, single-centre study. Eur J Haematol 2017;98:38-43.

20. Evens AM, David KA, Helenowski I, Nelson B, Kaufman D, Kircher $\mathrm{SM}$, et al. Multicenter analysis of 80 solid organ transplantation recipients with post-transplantation lymphoproliferative disease: outcomes and prognostic factors in the modern era. J Clin Oncol 2010;28:1038-1046. 